\title{
Identificação de Serviços a partir da Modelagem de Processos de Negócio
}

\author{
Leonardo Guerreiro Azevedo ${ }^{1,2}$, Fernanda Araujo Baião ${ }^{1,2}$, Flávia Santoro ${ }^{1,2}$, \\ Jairo Souza $^{1}$, Kate Revoredo ${ }^{1}$, Vinícios Pereira ${ }^{1}$, Isolda Herlain ${ }^{3}$ \\ ${ }^{1}$ NP2Tec - Núcleo de Pesquisa e Prática em Tecnologia \\ Universidade Federal do Estado do Rio de Janeiro (UNIRIO) \\ ${ }^{2}$ Departamento de Informática Aplicada - DIA \\ Universidade Federal do Estado do Rio de Janeiro (UNIRIO) \\ ${ }^{3}$ Petrobras - Petróleo Brasileiro S/A \\ \{azevedo, fernanda.baiao, flavia.santoro\} @uniriotec.br, \\ \{jairofsouza, katerevoredo, viniciospereira\}@gmail.com, \\ iherlain.POLITECepetrobras.com.br
}

\begin{abstract}
Various approaches for services development in SOA propose business processes as a starting point. However, there is a lack of service identification systematic methods during business analysis. Also, existing approaches typically rely on analysts and developers expertise, which consider one business process at a time for service derivation. We argue that there has to exist a holistic view of organizational business processes for an effective SOA approach to occur. This work proposes a heuristic-based method for identifying a set of candidate services to support business activities.
\end{abstract}

Resumo. Diversas abordagens para desenvolvimento orientado a serviços têm processos de negócio como ponto de partida. No entanto, as pesquisas não detalham sistemáticas de identificação de serviços a partir de modelos de processos de negócio. Assume-se que os processos são automatizados e que destes derivam-se os serviços. Entretanto, propomos um passo anterior, que corresponde ao entendimento dos requisitos a partir de todo o conjunto de processos de negócio de uma organização. Este artigo propõe um método para identificação de serviços adequados para apoio às atividades do negócio da organização.

\section{Introdução}

A implantação de SOA (Service-Oriented Architectures) em uma organização apresenta uma série de desafios, incluindo análise de aplicações de software; implantação de dispositivos de hardware, servidores, infra-estrutura de ESB (Enterprise Service Bus) e mecanismos de segurança; integração de aplicações com processos de negócio, sendo ainda necessário definir processos para modelagem, projeto, monitoramento e gestão de serviços [Papazoglou et al. 2007].

O ciclo de vida da engenharia de software tradicional não se aplica diretamente a SOA. Novos papéis arquiteturais e novas tarefas de desenvolvimento são introduzidos para tratar as características de desenvolvimento orientado a serviços [Gu e Lago 2007]. Diversos autores concordam que uma abordagem sistemática para desenvolvimento de 
serviços é fundamental [Papazoglou et al. 2007][Gu e Lago 2007][Erl 2005]. Nas abordagens identificadas, o ponto de partida são os processos de negócio. [Klückmann 2007] e [Josuttis 2007] destacam a necessidade do conhecimento dos processos de negócio, sendo uma tendência a identificação de serviços a partir de seus modelos.

Apesar desta tendência, não existe um consenso quanto ao melhor método de análise do negócio para especificação dos serviços. Observa-se que, na maioria das propostas, assume-se que os processos são automatizados e que destes derivam-se os serviços. Entretanto, a definição de processos automatizados não é suficiente. Argumentamos que existe a necessidade de uma visão integrada dos processos de negócio da organização, onde cada processo de negócio é derivado da cadeia de valor. Esta visão integrada é determinante para uma abordagem efetiva de identificação de serviços.

Este artigo trata do desafio da identificação do conjunto mais adequado de serviços para apoio às atividades do negócio da organização na gestão do ciclo de vida de serviços. Um método baseado em heurísticas é proposto.

Este trabalho está dividido da seguinte forma. A seção 1 corresponde a presente introdução. Na seção 2, são apresentados trabalhos relacionados e a motivação para o método proposto em um ciclo de vida de serviços. A seção 3 apresenta diretrizes para a identificação de serviços a partir de modelos de processos de negócio, enquanto que a seção 4 detalha o método proposto. Na seção 5 é apresentada a avaliação do método e, na seção 6, são destacadas as conclusões e trabalhos futuros.

\section{Ciclo de vida de serviços}

Modelos de ciclo de vida de serviços apresentam novos desafios quando comparados com os modelos tradicionais da engenharia de software, por exemplo: como alinhar requisitos de negócio com soluções de TI em SOA, como manipular serviços distribuídos além dos limites organizacionais de maneira segura, como tratar versões de serviços de acordo com mudanças nos processos de negócio. Dessa forma, um modelo de ciclo de vida é vital para o bom funcionamento de SOA [Pulier e Taylor 2006].

[Gu e Lago 2007] afirmam que não há consenso para um modelo de ciclo de vida de serviços na literatura. Estes autores avaliaram uma série de propostas e constataram que as seguintes características são importantes: apresentar uma seqüência bem definida de passos com um bom nível detalhamento, estes passos estarem dividido nos três estágios de ciclo de vida (projeto, execução e mudança), além de terem suas atividades associadas a papéis que serão responsáveis por executarem estas atividades.

Entretanto, propostas apresentadas na literatura não descrevem em um nível de detalhes adequado a atividade de identificação de serviços a partir da modelagem de processos. Por exemplo, [Erl 2005] apresenta um passo-a-passo para modelagem de serviços, todavia, não é proposto um método estruturado para identificação de serviços. [Papazoglou e Heuvel 2006] apresentam princípios para identificação de serviços, tais como baixo acoplamento, alta coesão e nível de granularidade, sem detalhar os passos necessários. [Marks e Bell 2006)] ressaltam o reuso como princípio para identificação de serviços. [McBride 2007] sinalizam que, a partir de um requisito de negócio, deve-se buscar, no repositório de serviços, serviços que atendam aos requisitos de negócio ou que possam ser modificados para atender ao requisito de negócio, ou novos serviços devem ser implementados. [Jamshidi et al. 2008] consideram modelos de processos de 
negócio da organização e modelos de entidade da organização e propõem uma abordagem para identificação e especificação de serviços. A abordagem assume que o modelo de processo de negócio é altamente detalhado (até no nível de processo de negócio elementar, ou EBP (Elementary Business Process), e que a granularidade de cada entidade do negócio deve estar no mesmo nível do EBP que a cria. Modelos de processos neste nível de abstração não são fáceis de serem elaborados, podendo demandar um tempo considerável. Além disso, esta proposta não considera os elementos de um processo de negócio, tais como regras de negócio, requisitos de negócio, fluxos do processo e funções recorrentes em fluxos de processo.

Portanto, identificar os serviços que atendam a requisitos de negócio específicos é totalmente dependente do conhecimento e memória do analista SOA. De maneira geral, a literatura aponta para a importância de se analisar quais funcionalidades dos processos devem ser expostas como serviços ou implementadas em aplicações. Todavia, decidir por um serviço que atenda a um requisito não é trivial, sendo necessário um método detalhado para identificação de serviços durante a análise do negócio. Uma proposta é apresentada na Seção 3.

\section{Diretrizes para a identificação de serviços a partir de modelos de processos de negócio}

A identificação de serviços a partir de processos de negócio modelados tem o objetivo de responder às seguintes questões:

- como definir o escopo de processos para identificar serviços?

- como identificar os serviços mais adequados para atender ao escopo pré-definido?

- quais elementos utilizados na modelagem de processos devem ser considerados nesta identificação?

- que tipos de serviços podem ser identificados a partir da modelagem de processos?

Esta seção apresenta diretrizes para identificação de serviços candidatos a partir da modelagem de processos de negócio. Serviço candidato é uma abstração (não implementada) de serviço a qual, durante a fase de projeto em um modelo de ciclo de vida de serviço, pode ser escolhida para ser implementada como um serviço ou como uma funcionalidade de uma aplicação [Erl 2005].

\subsection{Modelagem de Processos de Negócio}

A modelagem de processos de negócio compreende a construção de um conjunto de visões integradas que proveem entendimento comum do negócio (Figura 1). Existem várias abordagens para modelagem de negócio, e cada uma delas define diferentes notações e linguagens de modelagem. Independente da abordagem utilizada, os seguintes modelos podem ser considerados pelos os analistas para identificação de requisitos:

1) Modelo Organizacional: corresponde às unidades organizacionais, papéis e relacionamentos;

2) Modelo de Objetivos: mostra as metas estratégicas e como são divididas em submetas; 
3) Modelo de Processos: representa os macroprocessos de negócio e fluxos de atividades que compõem cada processo;

4) Modelo de Atividades: representa o relacionamento entre as atividades e os responsáveis por cada uma delas, além de detalhes tais com objetos e informações manipuladas, regras de negócios e requisitos de negócio [Sharp e McDermott 2001].

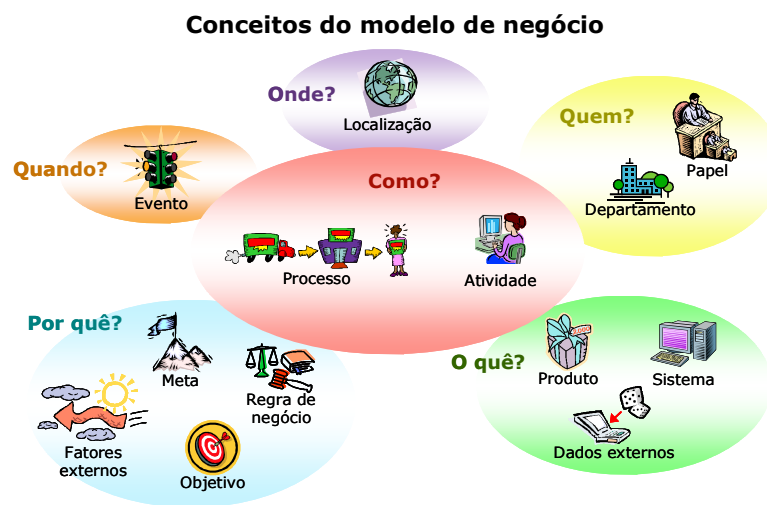

Figura 1. Perspectivas dos modelos de negócios.

Um dos aspectos mais importantes na modelagem do negócio é a explicitação das regras do negócio. Regras de negócio correspondem a declarações de políticas ou condições que devem ser satisfeitas pelos processos da organização e definem ou restringem alguns aspectos do negócio [Guide Business Rule Project 2008]. Regras de negócio são utilizadas para determinar a dependência entre atividades, explicitando o fluxo dos processos de negócio, ou para explicitar regras específicas de uma atividade do processo. Os seguintes tipos de regras podem ser considerados: assertiva estrutural (definição de termo de negócio, relacionamento entre termos), restrição (integridade, condição ou autorização), derivação (cálculo matemático ou inferência).

Outro elemento fundamental é o requisito de negócio, que representa uma necessidade do negócio a ser apoiada por sistema. Os requisitos podem ser identificados durante a análise do processo como forma de melhoria dos mesmos. Portanto, requisitos de negócio podem constar em um modelo de processos futuro (modelo TO-BE) para serem implementados, ou em modelos atuais (modelos AS-IS) como documentação de sistemas de apoio existentes.

\section{O método proposto para identificação de serviços}

Um método para identificação de serviços deve ter como ponto de partida uma demanda de desenvolvimento de software. Esta demanda deve ser descrita por um conjunto de requisitos que serão suportados por componentes de software, onde cada componente pode ser implementado como um serviço ou como funcionalidades de uma aplicação.

Em seguida, os modelos de processos que estejam relacionados à demanda devem ser analisados a fim de identificar serviços candidatos. Destes modelos de processos, devem ser analisadas as atividades e os fluxos que incluam atividades automáticas, automatizáveis e apoiadas por sistemas, as quais serão utilizadas nas etapas subsequientes. Atividades manuais (que não estão consideradas para automatização pela demanda) são descartadas, pois não faz sentido desenvolver serviços para as mesmas. 
De acordo com o detalhamento de informações existentes nos modelos, os serviços candidatos identificados podem ser classificados em (i) de negócio, quando o serviço encapsula uma regra de negócio; (ii) de dados, quando o serviço executa apenas operações de manipulação de dados (Create, Retrieve, Update ou Delete); ou (iii) utilitário, quando o serviço implementa uma função recorrente em diversos processos de negócio, de vários domínios. Além disso, o método identifica serviços candidatos que podem ser compostos por outros serviços candidatos. Uma composição pode ser implementada como uma orquestração dos serviços (onde um serviço invoca os outros serviços componentes) ou como um serviço único contendo métodos que implementam as funcionalidades dos serviços componentes. $\mathrm{O}$ método proposto, no entanto, não trata da implementação dos serviços físicos,. Esta é uma decisão da fase de projeto de serviços, já que são necessárias informações como capacidade do hardware, largura de rede, etc.

O método de identificação de serviços proposto inclui os seguintes passos (Figura 2): (1) Seleção das atividades; (2) Identificação e classificação dos serviços candidatos; (3) Consolidação dos serviços candidatos.

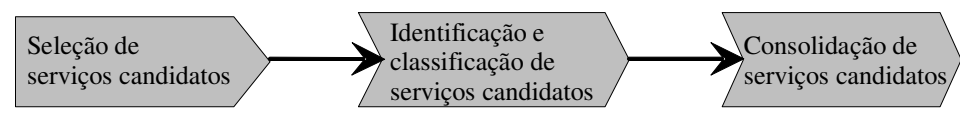

Figura 2. Método para idenficação de serviços.

A identificação de serviços a partir da modelagem de processos de negócio é um cenário que requer avaliação de um número muito grande de possíveis soluções. Neste caso, uma alternativa é a adoção de métodos heurísticos de busca onde soluções são buscadas por aproximações sucessivas, avaliando-se os resultados alcançados. As heurísticas propostas são definidas a fim de tratar tanto a análise sintática como a análise semântica de modelos de processos.

A análise sintática dos modelos de processos é realizada considerando a estrutura do modelo de processo. Portanto, nós propomos heurísticas para identificação de serviços para cada padrão de workflow proposto por [Van der Aalst 2000 e 2003]. Um padrão de workflow é uma abstração de uma forma concreta que ocorre repetidamente em contextos específicos. Os padrões de workflow de [Van der Aalst 2003] são sempre utilizados como benchmark para avaliar funcionalidades de sistemas de gestão de workflow. Ao considerarmos a especificação de padrões de workflow para a identificação de serviços, nós afirmamos estar cobrindo todas as possibilidades de fluxos de atividades que podem ser representadas por um modelo de processo.

A análise semântica de um modelo de processo para identificação de serviços deve considerar todas as indicações para automação de processos, seja total ou parcial. Dentre todos os elementos possíveis em um modelo de processos [Sharp e McDermott 2001], o método considera requisitos de negócio e regras de negócio em especial, dado que suas semânticas indicam funcionalidades que devem ser implementadas em serviços de apoio ao processo. Portanto, nós propomos heurísticas para identificação de serviços candidatos a partir de cada um destes dois elementos, quando ele está associado às atividades selecionadas na fase 1 .

O método de identificação proposto recebe como entrada a demanda de desenvolvimento de software, os modelos de processos TO-BE, e uma lista de requisitos 
de negócio que já estão implementados em aplicações existentes relacionadas com a demanda. O resultado final do método é uma tabela e grafos de dependência entre serviços candidatos, que auxiliarão o arquiteto no projeto e construção dos serviços.

Etapa 1: Seleção das atividades. Na primeira etapa do método, é feita a seleção das atividades dos processos TO-BE, a partir das quais podem ser identificados serviços candidatos. São selecionadas todas as atividades automáticas, automatizáveis ou apoiadas por sistemas.

Etapa 2: Identificação e classificação dos serviços candidatos. Na segunda etapa (Figura 3), as atividades selecionadas na etapa 1 são analisadas dentro dos seus contextos nos modelos de processos, segundo um conjunto de heurísticas que levam em consideração a semântica da atividade (regras de negócio, requisitos de negócio) e a estrutura do fluxo de atividades (padrões de workflow). As heurísticas propostas são descritas a seguir.

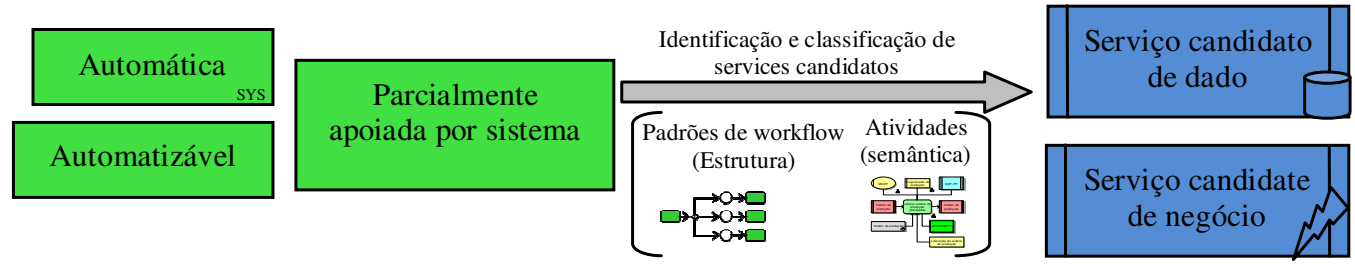

Figura 3. Etapa de identificação e classificação de serviços candidatos.

Heurística 1: Um serviço candidato deve ser identificado a partir de uma regra de negócio.

Exemplo: A partir da regra de negócio "Seleção de fornecedor de produto" com a descrição "Deve ser selecionado o fornecedor com as melhores condições comerciais referentes a preço e prazo" deve ser identificado o serviço candidato "Selecionar fornecedor de produto".

Heurística 2: Um serviço candidato deve ser identificado a partir de um requisito de negócio.

Exemplo: A partir do requisito de negócio "Consultar cotações de fornecedores", cuja descrição é "O sistema XPTO deve consultar as cotações de todos os fornecedores que fornecem determinado produto.", deve ser identificado o serviço candidato "Consultar cotações de fornecedores".

Heurística 3: Um serviço candidato deve ser identificado a partir de um conjunto de atividades seqüenciais.

Exemplo: A partir da seqüência de atividades "Comprometer limite de crédito", "Determinar taxa de juros a ser cobrada" e "Gerar contrato" (Figura 4), um serviço candidato deve ser identificado.

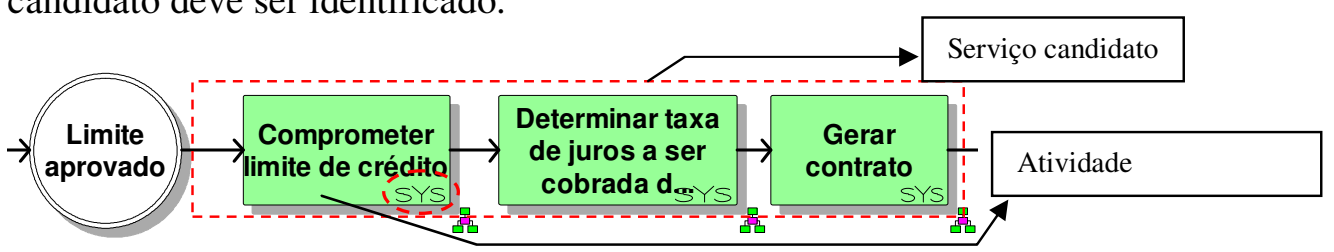

Figura 4. Exemplo de atividades seqüenciais. 
Heurística 4: Um serviço candidato deve ser identificado a partir de uma estrutura iniciada em um ponto no workflow onde um fluxo de controle simples divide-se em fluxos de controle múltiplos, que podem ser executados em paralelo, e finalizada em um ponto no workflow onde os múltiplos fluxos paralelos convergem em um fluxo de controle simples, sincronizando-os, ou onde ramificações terminem em evento final.

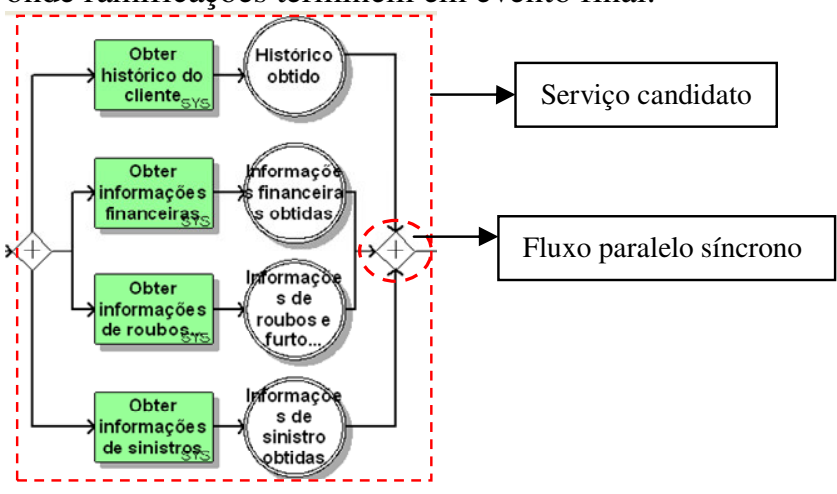

Figura 5. Exemplo de atividades que fazem parte de um fluxo AND.

Exemplo: Um serviço candidato deve ser identificado, a partir do fluxo descrito na Figura 5 para as atividades "Obter histórico do cliente", "Obter informações financeiras", "Obter informações de roubos e furtos" e "Obter informações de sinistros".

Heurística 5: Um serviço candidato deve ser identificado a partir de uma estrutura iniciada em um ponto no workflow onde, baseado em uma decisão, uma, e somente uma, de várias ramificações do fluxo é escolhida, e finalizada em um ponto no workflow onde as ramificações do fluxo se juntem sem sincronização ou quando uma ou mais das ramificações termina em evento final.

Exemplo: Um serviço candidato deve ser identificado para o fluxo representado pela Figura 6, correspondendo às atividades "Verificar situação cadastral do cliente" e "Atualizar informações do cliente", incluindo as regras de decisão.

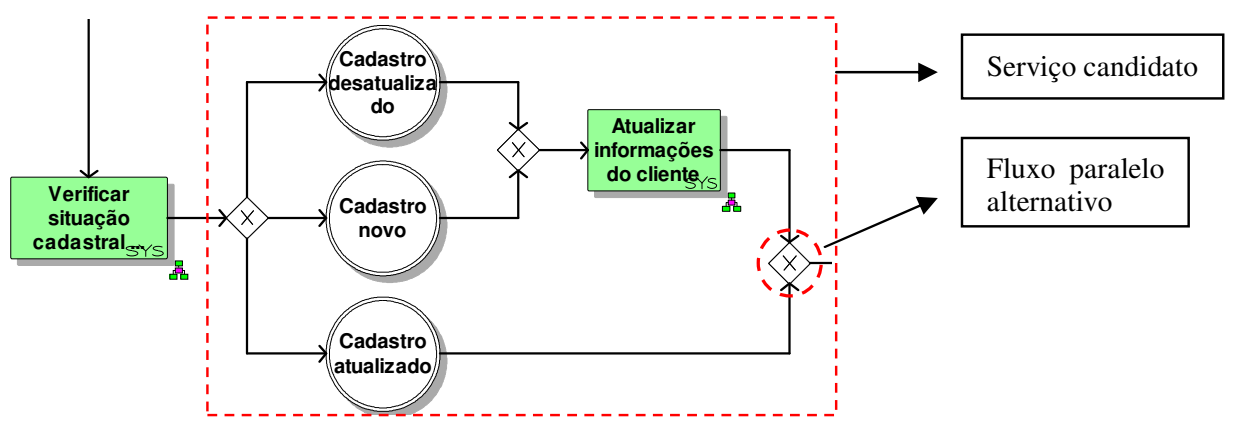

Figura 6. Exemplo de XOR.

Heurística 6: Um serviço candidato deve ser identificado a partir de uma estrutura iniciada em um ponto no workflow onde, baseado em uma decisão, uma ou mais ramificações do fluxo é escolhida e finalizada em um ponto no workflow onde as várias ramificações do fluxo se juntem. Se mais de um dos fluxos de origem foi executado, então é necessário sincronizá-los. Ramificações podem também terminar em um evento final.

Heurística 7: Um serviço candidato deve ser identificado a partir de uma estrutura do workflow onde uma ou mais atividades podem ser executadas repetidamente. 
Heurística 8: Um serviço candidato deverá ser identificado a partir de uma atividade que recebe uma mensagem enviada por uma atividade de outro workflow.

Exemplo: O processo "Tratar limites de mercadorias em estoque" (Figura 7.a), ao identificar que o limite de uma mercadoria em estoque foi atingido, envia um pedido de mercadoria para o processo "Fornecer mercadoria" (Figura 7.b). Segundo a "Heurística de interface de processo", um serviço candidato deve ser identificado para a atividade "Receber pedido de mercadoria".

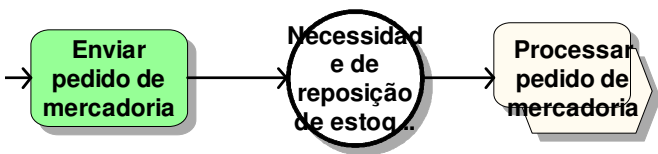

(a)

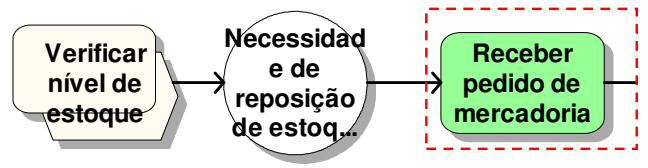

(b)

Figura 7. Exemplo de interface entre processos.

Heurística 9: Um serviço candidato deve ser identificado a partir de um ponto do workflow onde múltiplas mensagens são enviadas a partir de uma atividade para outras atividades conhecidas. Além do serviço remetente da mensagem, um serviço candidato deve ser identificado para cada destinatário. O serviço remetente é conhecido como broker e é responsável por receber a mensagem em questão e distribuí-la para todos os serviços destinatários. Existem serviços brokers que permitem que serviços destinatários inscrevam-se em sua lista de destinatários em tempo de execução. Isso faz com que a alteração dos destinatários seja transparente para uma aplicação cliente.

Exemplo: Na Figura 8, deve ser identificado um serviço para a atividade "Enviar solicitação de cotação" (serviço remetente) e um serviço para "Tratar solicitação de cotação" para cada fornecedor (serviços destinatários).

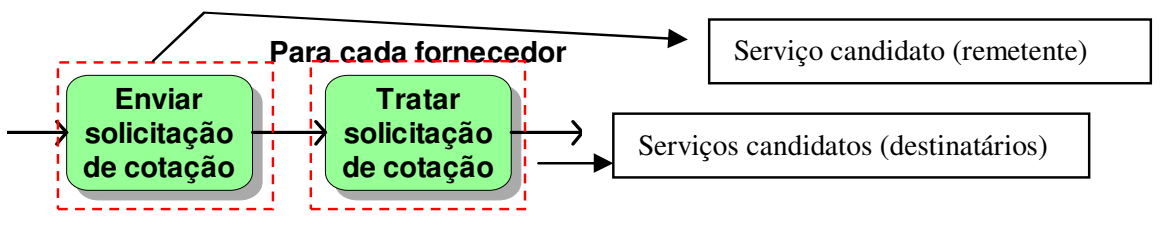

Figura 8. Exemplo de atividade de notificação e atividades de tratamento da notificação.

Etapa 3: Consolidação de Serviços Candidatos. Uma vez identificados os serviços candidatos, a consolidação tem por objetivo gerar, para cada serviço candidato, um conjunto de características que vão auxiliar a equipe de arquitetos SOA a definir a melhor forma de implementação do conjunto de serviços físicos. A consolidação é dividida nos seguintes passos:

1. Eliminar serviços duplicados;

2. Identificar grau de reuso para cada serviço: $O$ grau de reuso de um serviço candidato corresponde ao número de ocorrências da(s) atividade(s) da(s) qual(is) o serviço foi identificado. $\mathrm{O}$ grau de reuso é equivalente ao conceito de suporte, e pode ser calculado segundo [Agrawal et al., 1998];

3. Associar serviços candidatos aos sistemas que os implementam: Nos casos em que um serviço candidato já esteja implementado em algum sistema, deve-se registrar esta informação na tabela de consolidação. Esta informação permitirá ao projetista identificar os requisitos que podem ser expostos como serviços mais facilmente, reutilizando o código fonte. 
4. Associar os serviços candidatos aos requisitos da demanda: os serviços candidatos que foram identificados a partir da demanda devem ser associados à demanda. Esta indicação permitirá ao projetista identificar o conjunto mínimo de serviços necessários para atender à demanda inicial.

5. Associar serviços à(às) atividade(s) que lhe deram origem: Esta associação permite identificar todos os serviços que apóiam uma atividade, e pode-se com isso indicar a implementação de um serviço composto que encapsule todas as funcionalidades tratadas por esta atividade.

6. Identificar granularidade e dependência entre serviços candidatos: Os serviços candidatos devem ser associados aos serviços candidatos que os utilizam. Esta informação é obtida a partir do relacionamento entre regras de negócio e entre regras de negócio e requisitos de negócio, quando estes se encontram explícitos no modelo de processos. A associação entre serviços candidatos é uma representação da dependência entre eles, além de ser um indicador da granularidade de cada serviço candidato. Serviços candidatos que não utilizam nenhum outro serviço candidato são considerados de granularidade fina e são independentes de outros serviços. Serviços candidatos que utilizam outros serviços candidatos são de granularidade grossa, e são dependentes dos serviços que utilizam.

7. Identificar serviços utilitários: Deve-se marcar os serviços candidatos que foram identificados a partir de funções recorrentes em processos de negócio ou aspectos de estrutura organizacional como propostos por [Thom et al. 2007] como sendo serviços candidatos utilitários. A importância da análise de padrões baseados em fluxos recorrentes se dá porque, de acordo com [Thom et al., 2007], os processos de negócio freqüentemente incluem uma variedade de fragmentos que podem ser entendidos como atividades de bloco com semântica específica e bem definida. Em particular, um fragmento (ou função recorrente em processo de negócio) pode ocorrer diversas vezes em uma mesma definição de processo ou em modelos de processos diferentes. A Figura 9 apresenta um exemplo, onde a atividade "Verificar condições de contrato com cliente" corresponde ao "Padrão de Aprovação" [Thom et al. 2007] e pode ser generalizado como um serviço utilitário, ou seja, o serviço de aprovação de condições de contrato pode ser utilizado por diferentes papéis na organização de acordo com o nível hierárquico para aprovação de contrato de cliente.

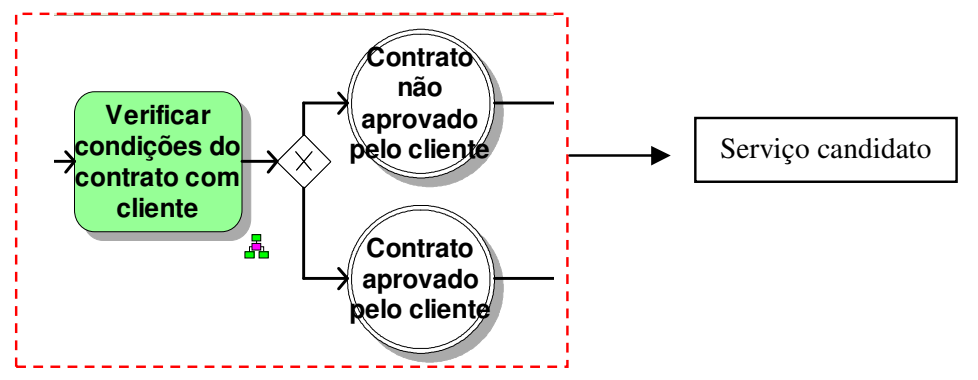

Figura 9. Exemplo de padrão de aprovação.

\section{Avaliação do método de identificação de serviços}

Com o objetivo de avaliar o método proposto, foi conduzido um estudo de caso na PETROBRAS, maior empresa brasileira de gás e petróleo. O método foi aplicado no 
processo "Realizar diagnóstico diário da produção de óleo", executando as três etapas propostas. $\mathrm{Na}$ execução da etapa 1, foram identificadas 19 atividades apoiadas por sistema ou automáticas. Em seguida, foi realizada a etapa 2, correspondente às heurísticas 1 a 9, a qual identificou 147 serviços candidatos. Na etapa 3, os serviços candidatos foram consolidados. As informações geradas por esta etapa para os serviços candidatos (grau de reuso, associação com sistemas, definição de granularidade inicial, dependência entre serviços) foram submetidas para avaliação por dois arquitetos da área de desenvolvimento de serviços PETROBRAS - TIC/TIC-E\&P/GDIEP, os quais inclusive já haviam implementado um conjunto de serviços de apoio ao processo em questão, a partir da identificação manual dos serviços necessários. Segundo os arquitetos, as informações de consolidação de serviços candidatos foram consideradas de grande utilidade, pois contém informações importantes para direcionar o projeto e construção de serviços. Ainda, segundo eles, o método proposto idenficou todos os serviços que haviam sido implementados.

As heurísticas que mais identificaram serviços candidatos foram as de regra e requisito de negócio, já que existia um amplo conjunto destes elementos explícitos no modelo de processos. Notou-se que a aplicação do método identificou tanto serviços de granularidade grossa quanto fina. Serviços gerados utilizando a Heurística 1 possuem, de maneira geral, granularidade fina, enquanto que a Heurística 2 e as baseadas em padrões de workflow podem gerar serviços candidatos de granularidade mais grossa. Em geral os serviços candidatos gerados a partir de padrões de workflow têm granularidade grossa, com exceção dos padrões relacionados a desvios de fluxo condicionais (conector XOR). Os casos de XOR encontrados têm mais uma atividade e um evento final além da atividade anterior ao XOR. Dessa forma, apenas a regra de negócio existente na atividade anterior ao conector deu origem a um serviço candidato, o qual tem granularidade fina.

Houve casos de serviços candidatos diferentes que correspondiam a uma mesma funcionalidade. Isso ocorre quando os serviços candidatos são gerados por heurísticas diferentes, que se baseiam em objetos diferentes do modelo de negócio, mas que dizem respeito a uma mesma funcionalidade. Percebeu-se que, para que esta identificação possa ser feita com confiabilidade, é preciso que o modelo explicite o relacionamento entre requisitos de negócio e suas regras de negócio correspondentes. Outro benefício do relacionamento explícito entre regras de negócio e requisitos de negócio é a conseqüente possibilidade de associação de serviços gerados a partir de regras de negócio com os requisitos recebido como demanda. Caso o modelo não permita este relacionamento explicitamente, os serviços candidatos gerados pela Heurística de regra de negócio não serão indicados como prioritários através da associação com a demanda, já que esta associação não é possível. As entradas e saídas dos serviços candidatos podem ser identificadas automaticamente, caso seja explicitado no modelo o relacionamento entre regras de negócio e as informações que são referenciadas por elas.

\section{Conclusão}

A implantação de SOA em uma organização apresenta uma série de desafios, dentre os quais destacamos questões relacionadas à modelagem, projeto, monitoramento e gestão de serviços. As propostas de modelos de ciclo de vida que estão no estado da arte da literatura são, em geral, abstratas, e não existe um consenso de um modelo de ciclo de vida de serviços. Um bom ciclo de vida de serviços não apenas facilita a gerência do 
ciclo de vida de sistemas orientados a serviço como também pode melhorar sua governança.

Neste trabalho, dentre as atividades do modelo de ciclo de vida, foi dada maior ênfase à atividade identificação de serviços a partir da modelagem de processos. Sendo assim, propomos uma análise top-down dos modelos de processos, identificando serviços a partir das regras de negócio, requisitos de negócio e de workflows. Como resultado destas atividades, um conjunto de serviços candidatos é identificado e consolidado quanto a aspectos como o grau de reuso do serviço, em quais sistemas o serviço está implementado, qual a relação do serviço candidato com a demanda de desenvolvimento, quais atividades estão relacionadas ao serviço, relacionamentos entre serviços e granularidade. $\mathrm{O}$ conjunto de serviços candidatos consolidado serve de entrada para o projeto de serviços, e auxilia o projetista a decidir se o serviço candidato deve ser implementado como serviço físico, como um componente de um serviço ou como um método de um componente.

A proposta de identificação de serviços foi avaliada em um estudo de caso sobre um processo de negócio de uma organização real na área de Óleo e Gás. A partir deste estudo de caso foram identificados 147 serviços candidatos para o modelo de processo considerado. $\mathrm{O}$ conjunto resultante de serviços candidatos foi considerado adequado por especialistas (analistas e desenvolvedores) de serviços.

Como trabalhos futuros, propomos a implementação de uma ferramenta para automatizar o método proposto, além da realização de estudos de caso para validação da proposta em outros domínios. Além disso, propomos o estudo de heurísticas para tratar dos critérios de projeto de serviços, tais como desempenho, granularidade mais adequada de serviço de acordo considerando características físicas (capacidade do hardware, largura de banda de rede, etc.), orquestração e composição de serviços.

\section{Agradecimentos}

Agradecemos à Petrobras, em especial à TIC/TIC-E\&P/GDIEP pelo apoio ao projeto do qual resultou a proposta deste trabalho.

\section{Referências}

Erl, T. (2005) "Service-Oriented Architecture: concepts, technology, and design", Prentice Hall.

Gu, Q., Lago, P. (2007) A stakeholder-driven service life cycle model for SOA. In 2nd international Workshop on Service Oriented Software Engineering: in Conjunction with the 6th ESEC/FSE Joint Meeting, pp 1-7, Croatia.

Guide Business Rule Project (2008) http://www.businessrulesgroup.org/first_paper/br01c1.htm\#s1e (acessado em agosto de 2008)

Jamshidi, P., Sharif, M., Mansour, S. (2008) "To Establish Enterprise Service Model from Enterprise Business Model". Proceedings of the 2008 IEEE International Conference on Services Computing, vol. 1, pp. 93-100.

Josuttis, N. (2007) “SOA in practice: The Art of Distributed System Design”. O'Reilly.

Klückmann J. (2007) Steps to Business-Driven SOA, ARIS Expert Paper, 
http://www.ids-scheer.com/set/6473/ARIS_Expert_Paper-SOA-

10_Steps_to_SOA_Klueckmann_2007-03_en.pdf (acessado em junho de 2008).

Marks, E. A., Bell, M. (2006), Service-Oriented Architecture: a planning and implementation guide for business and technology, John Willey \& Sons Inc.

McBride, G. (2007) The role of SOA quality management in SOA service lifecycle management. DeveloperWorks, Rational SOA Go to Market Manager, IBM. ftp://ftp.software.ibm.com/software/rational/web/articles/soa_quality.pdf. (acessado em agosto 2008).

Papazoglou, M. P., van den Heuvel, W. J. (2006) Service-oriented design and development methodology. Int. Journal Web Engineering and Technology (IJWET), vol. 2(4), p.412-442.

Papazoglou, M. P., Traverso, P., Dutsdar, S., Leymann, F. (2007) Service-Oriented Computing: State of the Art and Research Challenges. IEEE Computer Society, vol. 40 (11), p. 38-45.

Pulier, E., Taylor, H. (2006), Understanding Enterprise SOA. Manning.

Sharp, A., McDermott, P. (2001). Workflow Modeling: Tools for Process Improvement and Application Development, Artech House.

Thom, L., Iochpe, C., Reichert, M. (2007) Workflow Patterns for Business Process Modeling._In: B. Pernici, J.A. Gulla (Eds.): Proc. of the CAiSE'06 Workshops, vol. 1, 8th Int'l Workshop on Business Process Modeling, Development, and Support (BPMDS'07), Trondheim, Norway. p. 349-358.

Tsai, A., Jiacun, W. T. W., Rosea, D. (2006) EPC Workflow Model to WIFA Model Conversion. In Proceedings of IEEE International Conference on Systems, Man and Cybernetics 8, p. 2758-2763, Taipei.

Van der Aalst, W. M. P., Ter Hofstede, A. H. M., Kiepuszewski, B., Barros, A. P. (2000) Advanced workflow patterns. In Proceedings of the 7th International Conference on Cooperative Information Systems (CoopIS 2000), vol. 1901 of Lecture Notes in Computer Science, pp. 18-29.

Van der Aalst, W. M. P., Ter Hofstede, A. H. M., Kiepuszewski, B., Barros, A. P. (2003) Workflow patterns. In Distributed and Parallel Databases vol. 14(1), p.5-51. 